\title{
ANALYSIS OF SOLITON FISSION OVER A SUBMERGED STRUCTURE USING "NONLINEAR FOURIER TRANSFORM (NLFT)"
}

\begin{abstract}
Markus Brühl ${ }^{1}$ and Hocine Oumeraci ${ }^{1}$
When a single incident solitary wave passes over a submerged reef, it disintegrates into a train of solitons (soliton fission), followed by a train of oscillatory waves. One of the major problems in the analysis of the recorded time series is the uncertain identification of the number of solitons $\mathrm{N}_{\mathrm{sol}}$ in the transmitted wave train behind the reef due to the difficulties to distinguish between solitons and oscillatory waves, especially in the case of breaking waves. With the "nonlinear Fourier transform (NLFT)", an application of the inverse scattering transform (IST) of the Korteweg-de Vries equation, a powerful analysis method is proposed to analyse nonlinear wave processes. Application of the NLFT to the transmitted waves of systematic numerical tests with breaking and non-breaking solitary waves behind a submerged structure (reef) with finite width $b_{r}$ allows to separate distinctly solitons and oscillatory waves. The paper gives an overview over the first NLFT analysis results for the determination of the number of solitons $N_{\text {sol }}$ behind the reef. The influence of relative submergence depth $d_{r} / h$, relative reef width $b_{r} / L_{i}$ and relative incident wave height $H_{i} / d_{r}$ is examined. First recommendations for the distinct identification of the number of solitons arising from the fission using NLFT will also be provided, including the limitations of the method.
\end{abstract}

Keywords: solitary wave, soliton fission, submerged reef, nonlinear Fourier transform, inverse scattering transform

\section{INTRODUCTION}

The processes of wave transformation over submerged reefs with finite width have been extensively studied for regular and irregular waves (e.g. Bleck \& Oumeraci, 2002) as well as for solitary waves (e.g. Strusinska \& Oumeraci, 2008), because they are important for a proper assessment of the hydraulic performance of submerged wave damping structures. For solitary waves only the propagation over submerged reefs with infinite width has been systematically investigated.

When a single solitary waves passes over a submerged reef with infinite width, due to the sudden strong change of the water depth, it becomes a so-called undular bore that will disintegrate into a train of solitons with smaller wave heights (soliton fission), followed by a train of oscillatory waves. These waves, due to their reduced wave energy, can better be handled in terms of shore protection.

Since 2007 experimental small-scale model tests with artificial submerged reefs with finite width have been performed at Leichtweiß-Institute (LWI) (Strusinska \& Oumeraci, 2008). The purpose of these tests was to identify the number of transmitted solitons behind the reef, their wave heights and to determine a design formula that explicitly considers the finite reef width.

One of the major problems during the solitary wave analysis was the reliable distinction of transmitted solitary waves and trailing oscillatory waves from the wave gauge recordings behind the reef, especially in the case of breaking waves.

Since 2008, a "nonlinear Fourier transform" (NLFT) is under implementation at LWI that explicitly considers solitons in the analysis algorithm and is able to reliably identify them in the original data. Applying this advanced powerful analysis method to the data of transmitted waves will help to clearly identify the number of solitons $N_{\text {sol }}$ behind the reef and to get reliable data for the development of a design formula. By using a numerical RANS/VOF model (COBRAS-UC), the range of reef widths could be increased, so that the influence of the reef width $b_{r}$ on $N_{\text {sol }}$ can be determined. Finally, an approach for the determination of the expected number of solitons behind submerged reefs with finite width can be developed.

\section{SOLITON FISSION OVER SUBMERGED REEFS}

The interactions between solitary waves and obstacles with infinite width such as long slopes or coastal shelfs have been described e.g. by Madsen and Mei (1969), Losada et al. (1989) and SeabraSantos et al. (1987). Especially the influence of the relative submergence depth $d_{r} / h$ (with $d_{r}$ the submergence depth and $h$ the water depth in front of the obstacle) that is the only governing parameter for the prediction of the number $N_{\text {sol }}$ of transmitted solitons over the obstacle is determined. Furthermore, the modification of the wave profiles, the type of wave breaking and the energy balance (reflected, transmitted and dissipated energy) are described. Numerical studies by Lin (2004) compared

\footnotetext{
${ }^{1}$ Leichtweiß-Institute for Hydraulic Engineering and Water Resources (LWI), Technische Universität Braunschweig, Beethovenstraße 51a, 38106 Braunschweig, Germany
} 
energy coefficients for reflection, transmission and energy dissipation of rectangular obstacles with finite and infinite widths. Detailed analyses for the influence of the reef width on the number of transmitted solitons behind submerged reefs are not available.

First, the process of soliton fission over reefs with infinite width will be discussed using the example given in Figure 1. An incident solitary wave with $H_{i}=0.22 \mathrm{~m}$ propagates in a water depth of $h=0.6 \mathrm{~m}$ towards a submerged reef with height $h_{r}=0.50 \mathrm{~m}$. At $t=10 \mathrm{~s}$ the solitary wave is in front of the reef. At $t=20 \mathrm{~s}$ the wave passed the reef front and is transformed in a bore that propagates over the reef. The front of the bore is highly turbulent. With increasing distance from the reef front, the fission process takes place and waves are generated at the front of the bore. If the reef length is long enough, a wave train of single (isolated) solitons will evolve from this so-called undular bore. Figure 2 shows the detailed water surface elevation of the soliton fission at the end of the simulation area $(x=100-$ $125 \mathrm{~m}, t=77.0 \mathrm{~s}$ ). We assume a soliton to be completely separated from the undular bore if the water surface elevation behind the soliton returns to still water level. Visual analysis of the data in Figure 2 shows that at least two solitons have already separated from the undular bore.
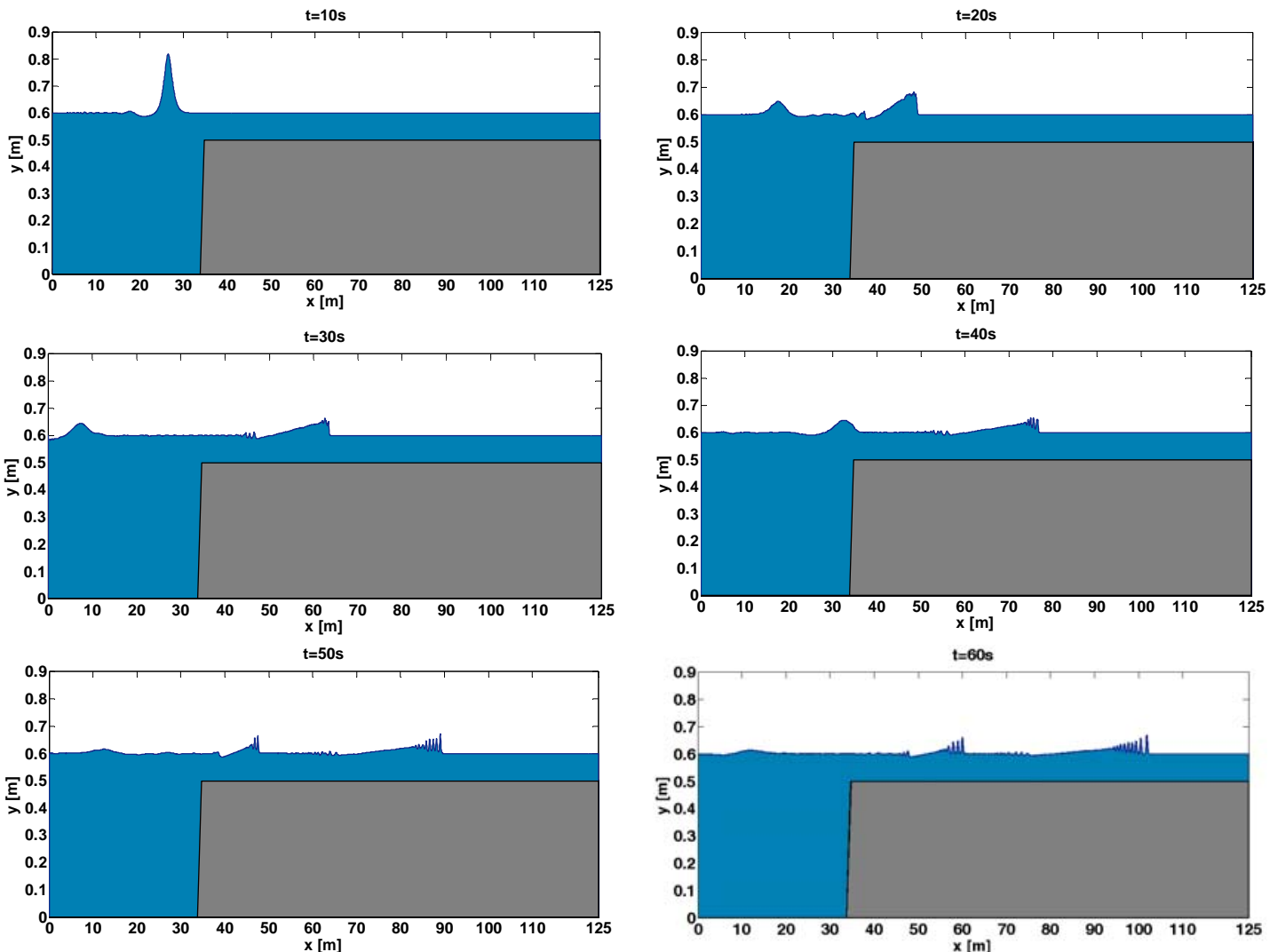

Figure 1. Fission of a solitary wave $\left(H_{i}=0.22 \mathrm{~m}\right)$ over a submerged reef $\left(h_{r}=0.5 \mathrm{~m}\right)$ with infinite width.

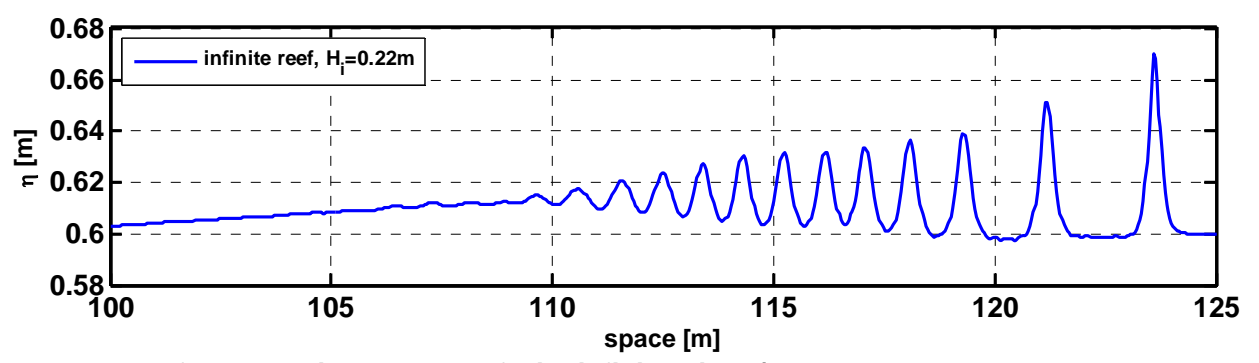

Figure 2. Water surface elevation over a reef with infinite width $\left(H_{i}=0.22 \mathrm{~m}, \mathrm{~h}=0.6 \mathrm{~m}, \mathrm{~h}_{\mathrm{r}}=0.5 \mathrm{~m}, \mathrm{~d}_{\mathrm{r}}=0.1 \mathrm{~m}\right)$.

Figure 1 shows at $t=20 \mathrm{~s}$ the reflected wave in front of the reef that in this case also is a soliton. It propagates towards the left boundary. The figure at $t=30 \mathrm{~s}$ shows the soliton after the reflection at the right side which is defined as closed boundary. This re-reflected solitary wave behaves in the same way as the incident soliton and finally becomes an undular bore over the reef. Depending on the wave 
properties and the structure geometry, the bore and the evolving solitons can be followed by oscillatory waves.

The expected number of solitons $N_{\text {sol }}$ over a reef with infinite width $b_{r}=\infty$ can be calculated by the following approaches:

Tappert and Zabusky (1971):

$$
\frac{\mathrm{d}_{\mathrm{r}}}{\mathrm{h}}=\left[0.5 \cdot \mathrm{N}_{\mathrm{sol}}\left(\mathrm{N}_{\mathrm{sol}}-1\right)\right]^{-4 / 9}
$$

Johnson (1972):

$$
\frac{\mathrm{d}_{\mathrm{r}}}{\mathrm{h}}=\left[0.5 \cdot \mathrm{N}_{\mathrm{sol}}\left(\mathrm{N}_{\mathrm{sol}}+1\right)\right]^{-4 / 9}
$$

Germain (1984) and Kabbaj (1984):

$$
\mathrm{M}=0.5\left\{\left[1+\frac{16\left(\mathrm{~h} / \mathrm{d}_{\mathrm{r}}\right)^{5 / 2}}{1+\left(\mathrm{h} / \mathrm{d}_{\mathrm{r}}\right)^{1 / 2}}\right]^{1 / 2}-1\right\}, \quad \text { with } \mathrm{N}=|\mathrm{M}| \text { the largest integer } \mathrm{N} \leq \mathrm{M} .
$$

According to these equations the expected number of solitons is between 9 and 11, followed by oscillatory waves. The equations do not consider the incident wave height $H_{i}$, the types of wave breaking or the reef width $b_{r}$.

Design formulae for the number of solitons behind submerged reefs with finite length are not available. The objective of the numerical simulations and analyses that are described here is to obtain first results for the number of transmitted solitons behind submerged reefs and get an improved insight into the governing processes. In this first approach the local processes at the beginning of the reef, such as the type of wave breaking, are assumed to be negligible for the number of transmitted solitons behind the reef.

\section{NONLINEAR FOURIER TRANSFORM}

The linear Fourier transform (FT) is the most common analysis method in frequency domain. The measured signal is decomposed into linear basic components (sine and cosine waves). Every basic component is represented in the Fourier spectrum by its characteristic wave parameters amplitude, frequency and phase. The linear superposition of all basic components provides the original signal. However, nearshore shallow water waves differ from linear sinusoidal shape and have nonlinear properties. This leads to mutual wave-wave interactions that can significantly affect the water surface profile which, in case of these wave-wave interactions, does not represent the linear superposition of the basic components any longer. Unfortunately, conventional wave analysis methods as the Fourier, wavelet or Hilbert-Huang transform cannot explicitly consider wave-wave interactions. In contrast, the advanced Nonlinear Fourier Transform (NLFT) explicitly considers these interactions during the analysis.

The initial point of the NLFT is the Korteweg-deVries (KdV) equation (Korteweg \& deVries, 1895) that describes the propagation of (cnoidal) waves in shallow water:

$$
\eta_{t}+c_{0} \cdot \eta_{x}+\alpha \cdot \eta \cdot \eta_{x}+\beta \cdot \eta_{x x x}=0
$$

The KdV equation can be solved exactly by the Inverse Scattering Transform (IST) (Ablowitz et al., 1973, 1974) for periodic boundary conditions by using cnoidal waves (Jacobian elliptic function) $\eta_{c n, j}(x, t)$. By using the so-called hyperelliptic functions $\mu_{j}(x, t)$ as basic components for the analysis, the cnoidal waves are extended by nonlinear wave-wave interaction terms (e.g. Osborne, 1991; Provenzale \& Osborne, 1991). Cnoidal waves represent the physical properties and shape of shallow water waves and include sinusoidal, Stokes and solitary waves, and thus one of the major limitations of conventional FT is overcome. With decreasing wave nonlinearity and decreasing nonlinear wave-wave interactions the hyperelliptic functions approximate the cnoidal waves. For the linear limit the hyperelliptic functions and cnoidal waves become sinusoidal and the NLFT reduces to the conventional FT. 
The hyperelliptic functions $\mu_{j}(x, t)$ can be decomposed into cnoidal waves $\eta_{c n, j}(x, t)$ and the nonlinear interaction term $\eta_{i n t, j}(x, t)$. The latter describes the modification of the surface profile that is caused by the nonlinear wave-wave interaction. The linear superposition of all cnoidal waves $\eta_{c n, j}(x, t)$ and its nonlinear interaction terms $\eta_{i n t, j}(x, t)$ provides the original data:

$$
\eta(x, t)=\sum \eta_{c n, j}(x, t)+\sum \eta_{\text {int }, j}(x, t)
$$

To illustrate the benefit of NLFT an example wave train is selected in Figure 3. Figure 4 shows the corresponding "nonlinear" Fourier spectrum of the selected data. The solitons are considered as basic components in the nonlinear analysis algorithm which are properly identified and represented in the "nonlinear" Fourier spectrum. The NLFT spectrum consists of the "soliton spectrum" with the soliton amplitudes $A_{j}$ (red bars) and the "radiation spectrum" (green line) which contains the amplitudes $a_{j}$ of the oscillatory waves (cosine and cnoidal waves). The soliton index $I_{j}$ (blue dashed line, also called modulus $m_{j}$ ) represents the nonlinearity of the determined basic components. For $I_{j}>0.99$ the waves are a solitons, for the limit $I=0$ the waves are sinusoidal. For $0<I_{j}<0.99$ the waves are cnoidal and the nonlinearity of these waves increases with $I_{j}$.

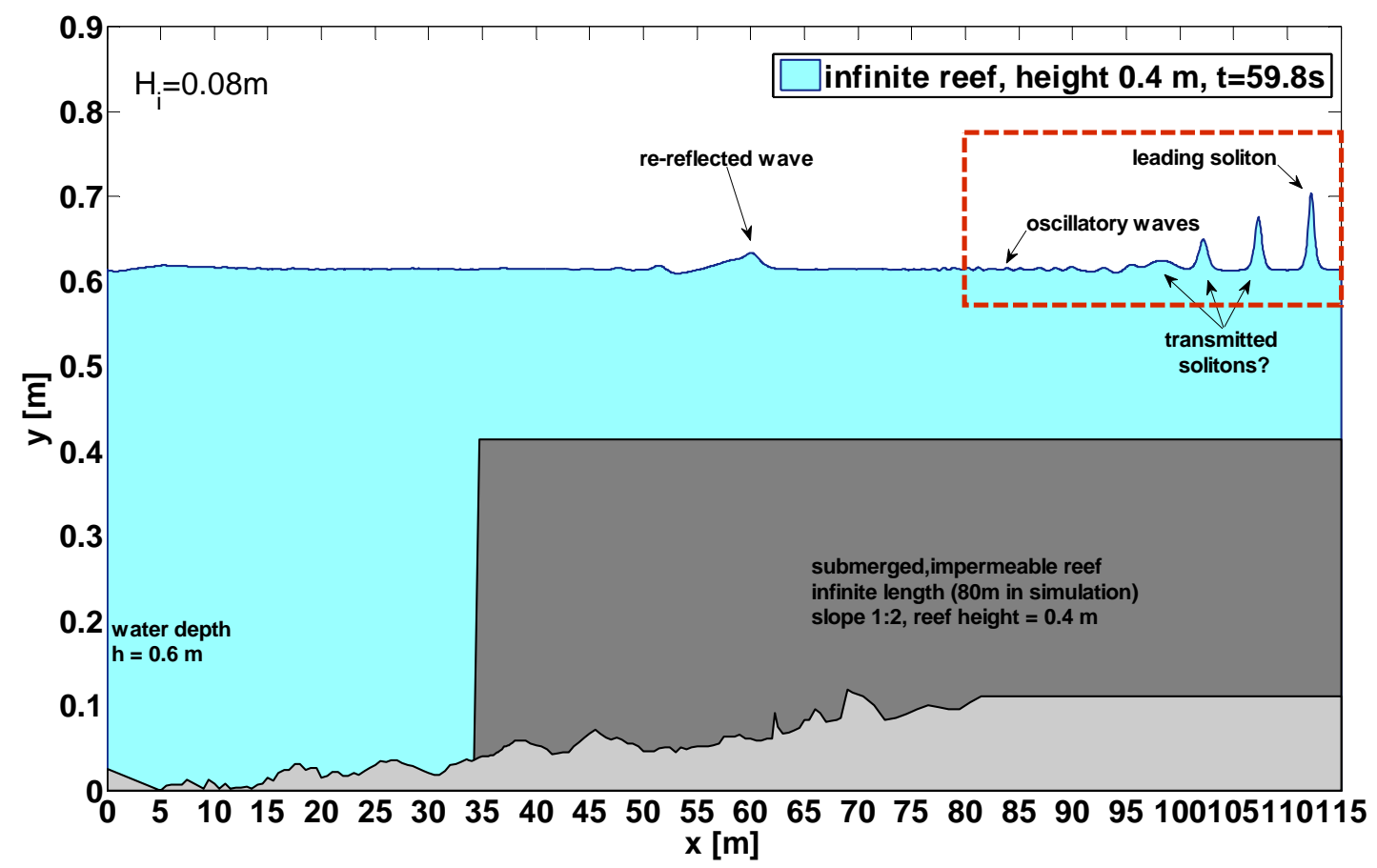

Figure 3. Selection of the example wave train with leading soliton, trailing solitons and oscillatory waves for the NLFT analysis.

Overall, the application of the NLFT on different space series of incident and transmitted waves showed that the "nonlinear" Fourier spectra are plausible and meet the expectations: (i) the obvious solitons (transmitted and reflected) are identified with correct amplitudes, (ii) the amplitudes of the oscillatory waves are small and (iii) the higher the wave number (i.e. shorter waves) the smaller the modulus $m$ and thus the nonlinearity of the waves.

The application of the NLFT to wave-structure interaction in shallow water and the analysis of this kind of numerical test data are unique. Reference data for numerical boundary conditions or program settings are not available and have to be determined empirically during the ongoing analyses. Comparative analyses with different numerical and artificially generated data have been performed with different settings for the resolutions of the wave numbers $k$ and the resolution $\Delta x$ of the data confirmed the sensitivity of the algorithm to these boundary conditions. Still, the application of NLFT to the wave-structure interaction is under development and many questions are not answered yet sufficiently. 


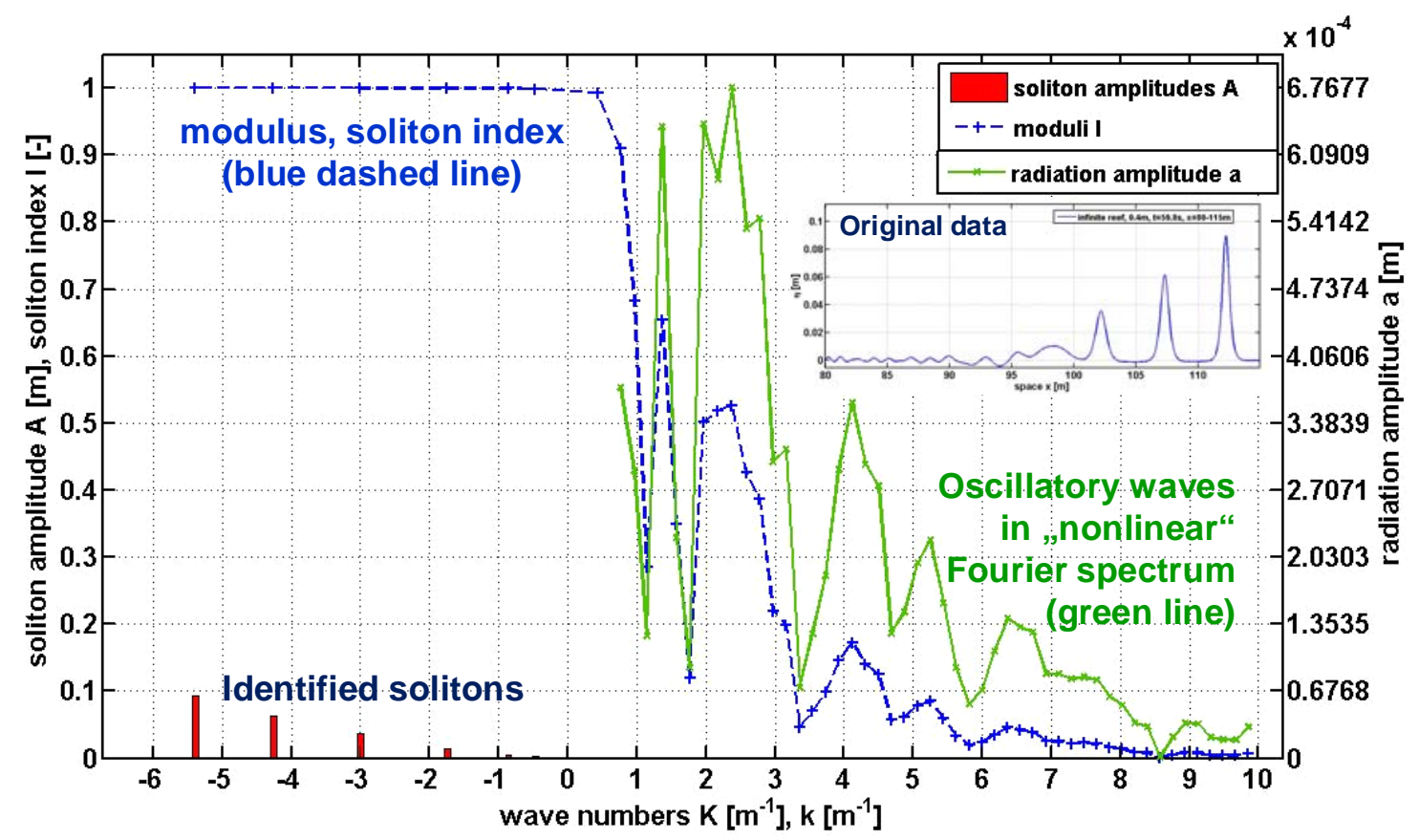

Figure 4. "Nonlinear" Fourier spectrum of the example wave train in Figure 3.

\section{NUMERICAL SET-UP, REEF GEOMETRIES AND TEST PROGRAM}

In 2007 and 2008 experimental small-scale model tests with solitary wave have been performed by Strusinska and Oumeraci (2008) in the small wave flume at LWI with reef widths $b_{r}=1.0 \mathrm{~m}$ and $2.0 \mathrm{~m}$. In 2010 a total number of 24 different numerical set-ups have been considered (see Figure 5), including those tested in the laboratory (Strusinska and Oumeraci, 2008) by varying the reef width $\left(b_{r}=2.0 \mathrm{~m}, 10.0 \mathrm{~m}, 30.0 \mathrm{~m}, 50.0 \mathrm{~m}, 70.0 \mathrm{~m}\right.$ and $\left.90.0 \mathrm{~m}\right)$ and the reef height $\left(h_{r}=0.30 \mathrm{~m}, 0.40 \mathrm{~m}\right.$ and $0.50 \mathrm{~m}$ ). The seaward reef top is located at $x=34.75 \mathrm{~m}$, the simulated total flume length is $125 \mathrm{~m}$. The reef with $b_{r}=90.0 \mathrm{~m}$ represents the "infinite" reef width and ranges until the end of the simulation area. Due to the variations of $h_{r}$ and the constant water depth $h=0.60 \mathrm{~m}$ for the submergence depth applies $d_{r}=0.10 \mathrm{~m}, 0.20 \mathrm{~m}$ and $0.30 \mathrm{~m}$. Figure 5 shows the set-up with three different reef heights $h_{r}$ and six different reef widths $b_{r}$.

The numerical simulations have been performed with the RANS/VOF model COBRAS-UC with incident solitary wave heights $H_{i}=0.08 \mathrm{~m}, 0.12 \mathrm{~m}, 0.18 \mathrm{~m}$ and $0.22 \mathrm{~m}$. The shape of the solitary wave is constant between the wave generation at the left boundary and the reef front. The total number of simulations with 18 set-ups and four wave heights is 72 .

The RANS/VOF model provides detailed information on the wave dynamics (horizontal and vertical velocities) around the reefs that are required for the analyses of the processes during the wavestructure interaction and for the validation of the NLFT analysis results. The NLFT analysis algorithm requires a resolution of $\Delta x=0.04 \mathrm{~m}$ in the space series that is provided by the simulations. The vertical mesh resolution is $\Delta y=0.015 \mathrm{~m}$.

\section{ANALYSIS OF SOLITON FISSION BEHIND SUBMERGED REEFS}

The first visual analysis of numerical simulations of wave-structure interaction between solitons and submerged reefs showed clearly that the processes of soliton fission (e.g. the locations at which the solitons separate from the undular bores and the total number of transmitted waves) depend on the incident soliton wave height $H_{i}$ and the submergence depth $d_{r}$. Therefore, in the first two steps, the influence of the incident solitary wave height $H_{i}$ on the development of solitons over the reef and the influence of the submergence depth $d_{r}$ over the reef are analysed. Third, the influence of the reef width $b_{r}$ on the number of solitons is determined. Finally, the results of the application of NLFT for the analysis of transmitted solitons $N_{\text {sol }}$ behind the submerged reefs with finite width are summarized and an outlook is given. 


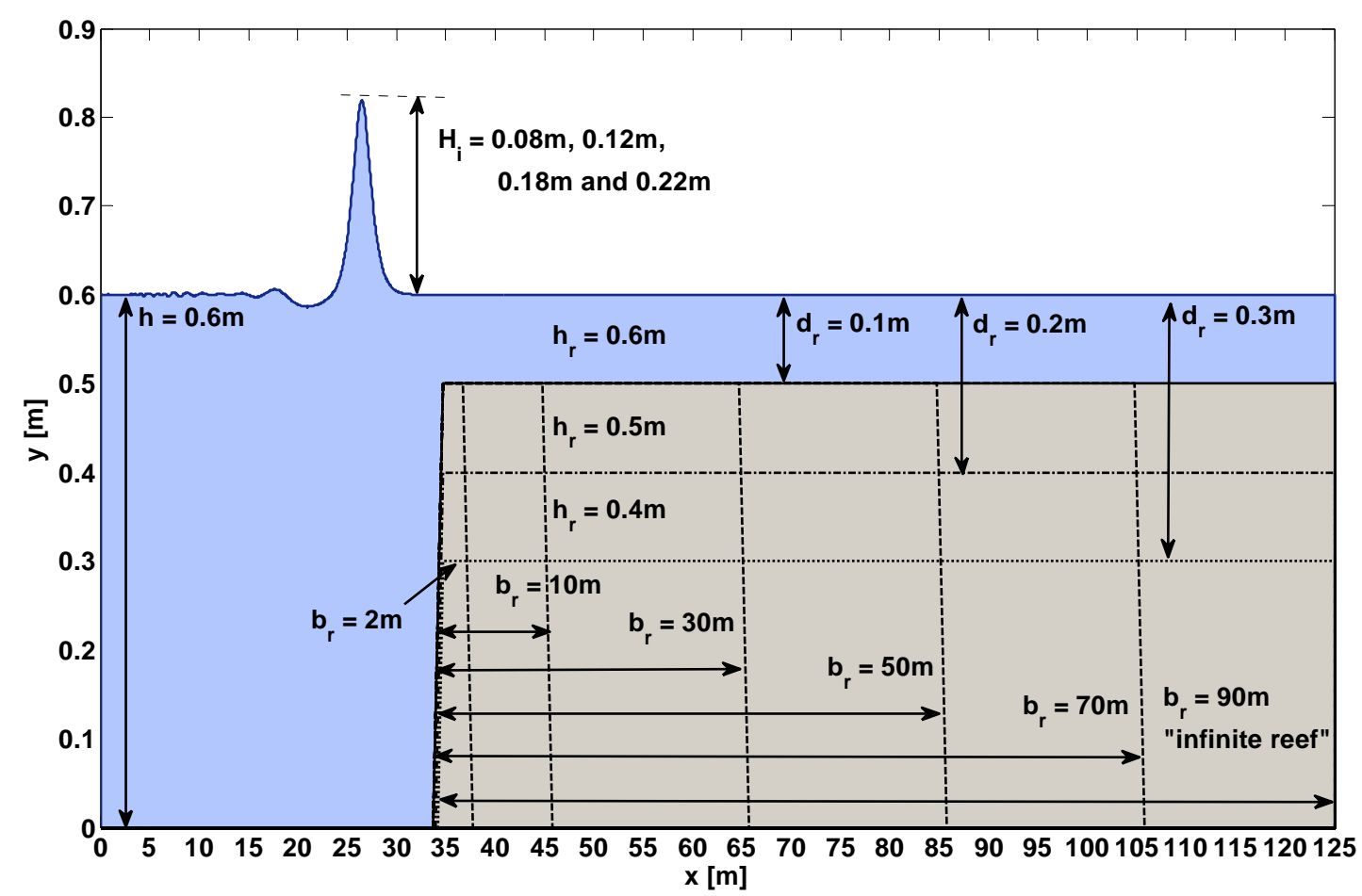

Figure 5. Numerical set-up for simulations with solitary waves over submerged reefs with finite width.

\section{Influence of incident solitary wave height $H_{i}$ on the development of solitons over reefs with infinite width}

Figure 6 shows in a) the incident solitary waves in front of the reef at simulation time $t=10.0 \mathrm{~s}$ in water depth $d=0.6 \mathrm{~m}$ and in b) their theoretical absolute and relative wave lengths. In Figure 6c) the nonlinear Fourier spectrum of the solitary wave with $H_{i}=0.22 \mathrm{~m}$ is given as an example for the nonlinear analysis of the incident waves. The red bar in the lower left corner represents the only soliton that is determined from the laboratory data. The wave height is correctly calculated with $H=0.22 \mathrm{~m}$. Similar spectra are available for the other incident waves. Due to the small waves that follow the solitons, a small number of oscillatory sinusoidal and cnoidal waves in the data with wave amplitudes $a<5 \cdot 10^{-3} \mathrm{~m}$ are determined.

For the analysis of the influence of the incident soliton wave height, the transmitted wave trains at the end of the "infinite" reef at $x=100-125 \mathrm{~m}$ are shown in Figure 7a). The space series clearly show that the analysed wave trains differ in the number of clearly isolated solitons and waves that have not separated yet. Due to the limited reef width of the "infinite" reef, the possible total number of solitons cannot be determined exactly. Nevertheless, the space series are analysed using Nonlinear Fourier Transform and the results compared. Figure 7c) shows the nonlinear Fourier spectra of the undular bores. The results clearly show that the NLFT is able to determine a large number of solitons from the undular bores, even if the solitons have not completely separated from the bore. Table 1 shows the number of solitons determined by i) visual analysis of the space series, ii) application of NLFT and iii) calculated from Equations (1) - (3). Furthermore, the number of undular waves in the bore is given. The total number of solitons and undular waves observed in the data corresponds very well to the number of solitons found from Equations (1) - (3). This confirms the assumption that the undular waves will disperse into separated solitons if the reef is long enough. In contrast to the visual observations the calculated number of solitons does not consider the incident wave height, although the observed solitons and the number of undular waves clearly show the influence of $\mathrm{H}_{\mathrm{i}}$. For $\mathrm{H}_{\mathrm{i}}=0.08 \mathrm{~m}$ the highest calculated soliton amplitudes from NLFT are $A=0.026 \mathrm{~m}, 0.022 \mathrm{~m}, 0.021 \mathrm{~m}, 0.020 \mathrm{~m}$, $0.018 \mathrm{~m}, 0.017 \mathrm{~m}, 0.015 \mathrm{~m}, 0.012 \mathrm{~m}$ and $0.008 \mathrm{~m}$. For $H_{i}=0.22 \mathrm{~m}$ the values are $A=0.077 \mathrm{~m}$, $0.056 \mathrm{~m}, 0.041 \mathrm{~m}, 0.037 \mathrm{~m}, 0.035 \mathrm{~m}, 0.034 \mathrm{~m}, 0.033 \mathrm{~m}, 0.031 \mathrm{~m}, 0.03 \mathrm{~m}, 0.028 \mathrm{~m}, 0.026 \mathrm{~m}$ and $0.024 \mathrm{~m}$. The analysis of undular bores and soliton wave trains with NLFT showed that the number of determined solitons is larger than the number of solitons that can be observed in the data. For data without bore, the number of solitons is determined more accurately. The reason is the shape of the turbulent bore, that does not correspond to cnoidal wave types. During the NLFT additional solitary 
waves are generated ("mathematical artefacts") that, if superposed and nonlinear interacting, provide the bore. This effect is similar to the additional sinusoidal wave components that are added in conventional FT if the analysed waves differ from sinusoidal shape.

a)

b)

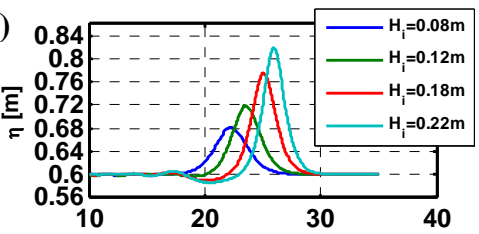

(

\begin{tabular}{|c|c|c|}
\hline $\mathrm{H}_{\mathrm{i}}[\mathrm{m}]$ & $\mathrm{L}_{\mathrm{i}}[\mathrm{m}]$ & $\mathrm{L}_{\mathrm{i}} / \mathrm{d}[-]$ \\
\hline 0.08 & 6.97 & 11.6 \\
\hline 0.12 & 5.69 & 9.5 \\
\hline 0.18 & 4.65 & 7.8 \\
\hline 0.22 & 4.20 & 7.0 \\
\hline
\end{tabular}

c)

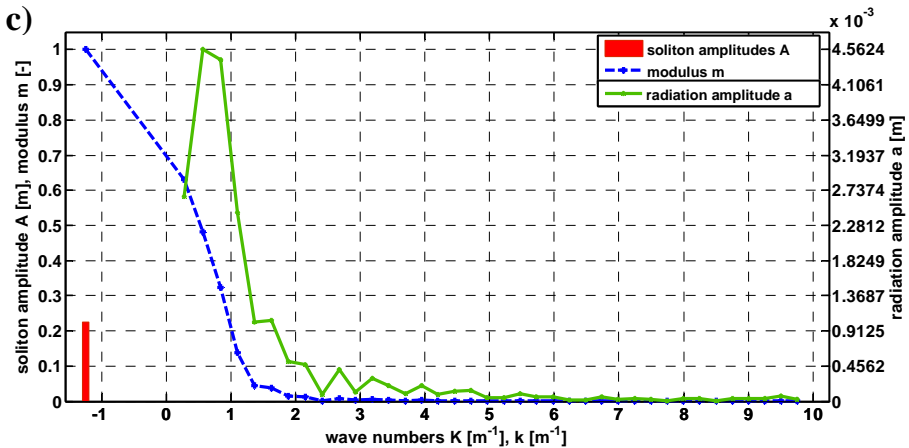

Figure 6. a) Incident solitary waves in front of the reef. Wave heights $H_{i}=0.08 \mathrm{~m}$ (blue line); $0.12 \mathrm{~m}$ (green); $0.18 \mathrm{~m}$ (red); $0.22 \mathrm{~m}$ (cyan). b) Absolute and relative wave lengths of incident solitary waves. c) Nonlinear Fourier spectrum of the incident solitary wave with $H_{i}=0.22 \mathrm{~m}$.

a)
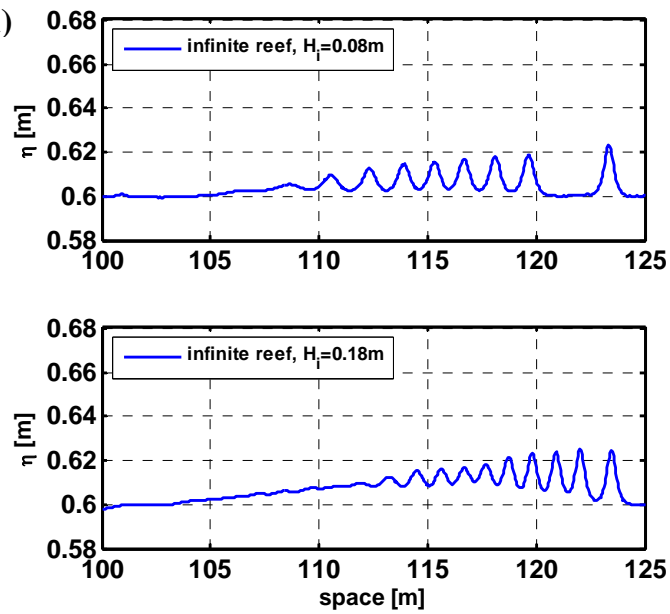
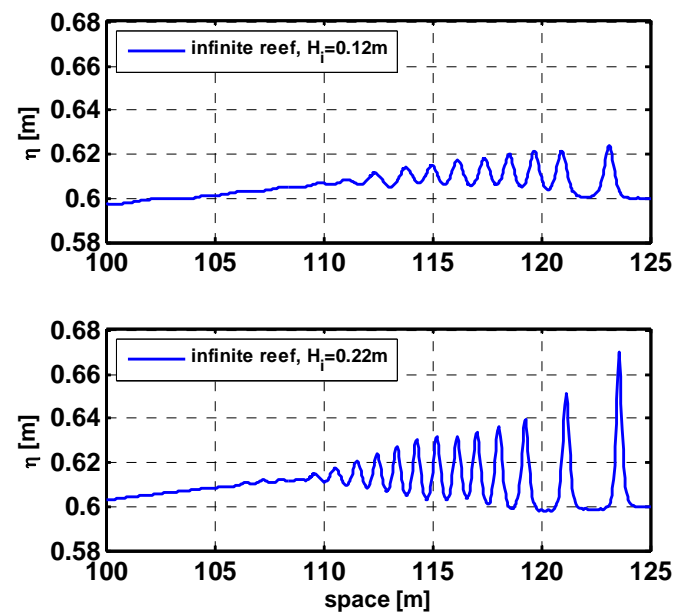

b)

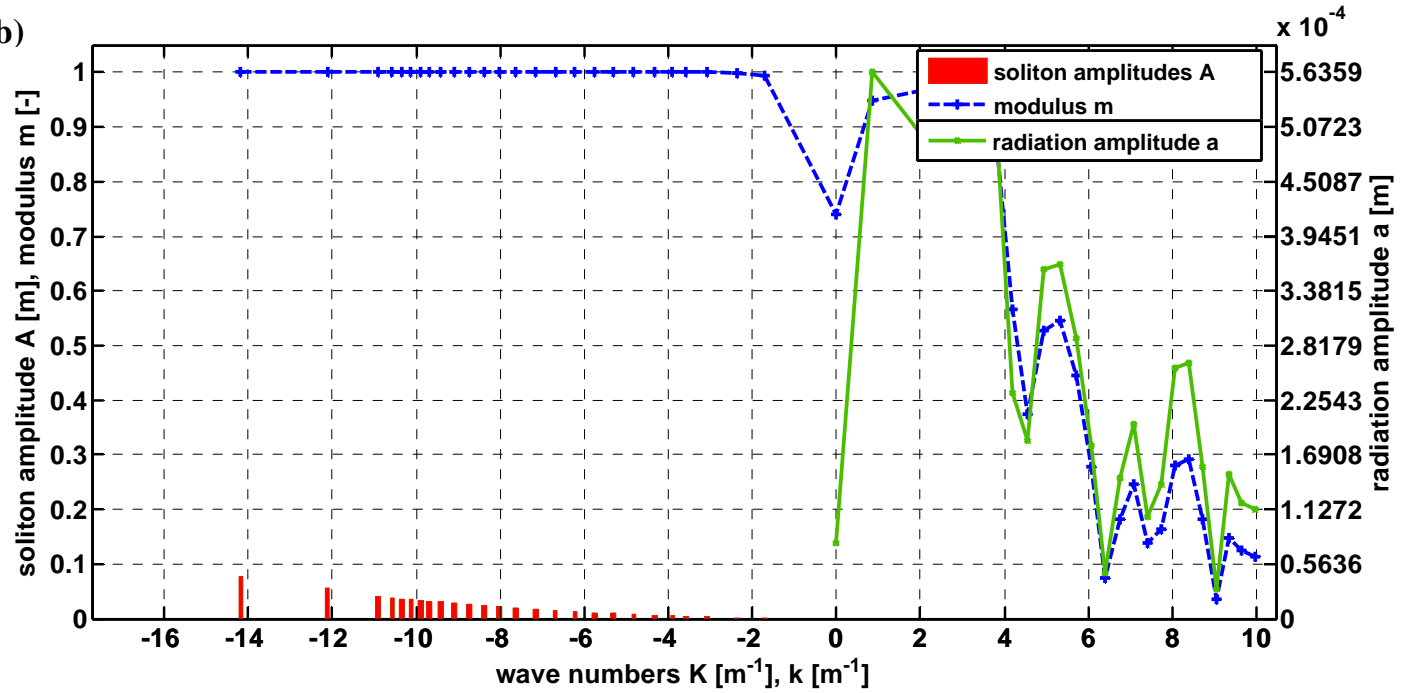

Figure 7. a) Transmitted wave trains at the end of the simulation area. b) Nonlinear spectrum of the wave train with $H_{i}=0.22 \mathrm{~m}$. 


\begin{tabular}{|c|c|c|c|c|c|c|}
\hline $\begin{array}{c}\text { wave } \\
\text { height } \\
H_{i}[\mathrm{~m}]\end{array}$ & $\begin{array}{c}N_{\text {sol }} \text { from } \\
\text { visual } \\
\text { observation - } \\
\text { data - } \\
\end{array}$ & $\begin{array}{l}\text { undular } \\
\text { waves at } \\
\text { front of } \\
\text { bore } \\
\end{array}$ & $\begin{array}{l}N_{\text {sol }} \text { from } \\
\text { NLFT } \\
\text { analysis }\end{array}$ & $\begin{array}{c}N_{\text {sol }} \text { by } \\
\text { Tappert and } \\
\text { Zabusky } \\
(1972)\end{array}$ & $\begin{array}{c}N_{\text {sol by }} \text { by } \\
\text { Johnson } \\
(1972)\end{array}$ & $\begin{array}{c}N_{\text {sol }} \text { by } \\
\text { Germain (1984) } \\
\text { and Kabbaj } \\
(1984)\end{array}$ \\
\hline 0.08 & 1 & 7 & 16 & \multirow{4}{*}{12} & \multirow{4}{*}{11} & \multirow{4}{*}{10} \\
\hline 0.12 & 1 & 8 & 21 & & & \\
\hline 0.18 & 1 & 9 & 22 & & & \\
\hline 0.22 & 2 & 10 & 26 & & & \\
\hline
\end{tabular}

For $H_{i}=0.08 \mathrm{~m}$ the visual observation of the data provides soliton amplitudes $A=0.023 \mathrm{~m}$, $0.019 \mathrm{~m}, 0.018 \mathrm{~m}, 0.017 \mathrm{~m}, 0.016 \mathrm{~m}, 0.015 \mathrm{~m}, 0.013 \mathrm{~m}, 0.009 \mathrm{~m}$ and $0.005 \mathrm{~m}$. The determined solitary wave amplitudes $A$ from NLFT and their relations correspond with the observed separated soliton and the undular waves, especially the small jumps in the wave height between solitons $1-2,7-8$ and 8 - 9 . The wave height is overestimated in the NLFT analysis by about $0.035 \cdot H_{i}$. For $H_{i}=0.22 \mathrm{~m}$ the analysed values are $A=0.070 \mathrm{~m}, 0.051 \mathrm{~m}, 0.039 \mathrm{~m}, 0.037 \mathrm{~m}, 0.034 \mathrm{~m}, 0.032 \mathrm{~m}, 0.032 \mathrm{~m}, 0.030 \mathrm{~m}$, $0.027 \mathrm{~m}, 0.024 \mathrm{~m}, 0.021 \mathrm{~m}$ and $0.017 \mathrm{~m}$. In this case, the soliton amplitude is overestimated by about $0.032 \cdot H_{i}$. This comparison shows that the NLFT does not distinguish between the solitons that have already separated from the undular bore and the waves inside the bore. The undular waves are regarded as solitons. Due to the close distance between them, they interact nonlinearly and the resulting wave heights in the signal are smaller than they would be if the solitons would have already separated. The nonlinear interaction terms reduce the visible wave height. The higher the waves, the stronger the nonlinear properties and the larger are the interactions. Therefore, the overestimation at $H_{i}=0.08 \mathrm{~m}$ is smaller than in the test with higher solitons. To check whether all the other solitons that are determined by the NLFT are mathematical artefacts, or whether these non-visible solitons will evolve from the bore if the reef is wide enough further analyses with more appropriate models would be required.

The comparison of simulation data with different incident wave heights over an infinite reef shows that the number of separated solitons at this location $(x=65-90 \mathrm{~m}$ behind the reef front, i.e. 13 to 21 times the incident wave length) can be determined from observation and by application of the available theoretical formulae. The NLFT determines the separated solitons, the clearly visible waves in the undular bore and an additional number of solitons that represent the rest of the bore. The number of solitons (observed and determined by NLFT) and of the waves in the undular bore at this location depends on the incident wave height.

\section{Influence of relative submergence depth $d_{r} / h$ over the reef on the development of solitons}

The relative submergence depth $d_{r} / h$ is the governing variable for the calculation of the number of expected solitons over reefs with infinite width. Figure 8 shows the transmitted wave trains for all incident wave heights $H_{i}$ and all reef heights $h_{r}$. Within the data for one $H_{i}$ the number of solitons decreases with increasing submergence depth $d_{r}$ (decreasing reef height $h_{r}$ ). Table 2 shows the number of visually observed solitons and the NLFT analysis results for the data in Figure 8. For reefs with $h_{r}=0.40 \mathrm{~m}$ and $0.30 \mathrm{~m}$ no undular bore can be identified in the data. For reef height $h_{r}=0.50 \mathrm{~m}$, the number of solitons and undular waves increases from 8 for $H_{i}=0.08 \mathrm{~m}$ to 9 for $H_{i}=0.12 \mathrm{~m}$ to 10 for $H_{i}=0.18 \mathrm{~m}$ and to 12 for $H_{i}=0.22 \mathrm{~m}$. For $h_{r}=0.40 \mathrm{~m}$ the data shows 5, 5, 6 and 7 solitons. For $h_{r}=0.30 \mathrm{~m}$ and $H_{i}=0.08 \mathrm{~m}$ only two clear solitons are found, followed by maybe a third one for $H_{i}=0.12 \mathrm{~m}, 0.18$ and $0.22 \mathrm{~m}$.

The results for $N_{\text {sol }}$ clearly show the influence of the bore on the number of solitons. Over reefs $h_{r}=0.4 \mathrm{~m}$ and $0.3 \mathrm{~m}$ no bore is generated and $N_{\text {sol }}$ from NLFT corresponds to the solitons observed in the data. Usually, the number of visually observed solitons is smaller than the number determined by NLFT, because in the observation solitons with very small amplitudes can hardly be distinguished from oscillatory waves and therefore, in case of doubt, are assumed to be oscillatory waves.

The observations and analyses confirm the strong influence of $d_{r}$ on the number of transmitted solitons. The results clearly show, that the physical processes over the reef and the process of bore generation change between $h_{r}=0.5 \mathrm{~m}$ and $0.4 \mathrm{~m}$. For submergence depth $d_{r}=0.2 \mathrm{~m}$ and $H_{i}=0.22 \mathrm{~m}$ the process of undular bore generation and separation of the solitons is much faster than observed over the higher reef. At $x=100-125 \mathrm{~m}$ all solitons have already evolved and the bore has disappeared. For $d_{r}=0.2 \mathrm{~m}$ and $H_{i}=0.08 \mathrm{~m}$ no undular bore is generated and the incident soliton disintegrates into 
several solitons directly at the beginning of the reef without forming a turbulent bore shape. The same applies for $d_{r}=0.30 \mathrm{~m}$.
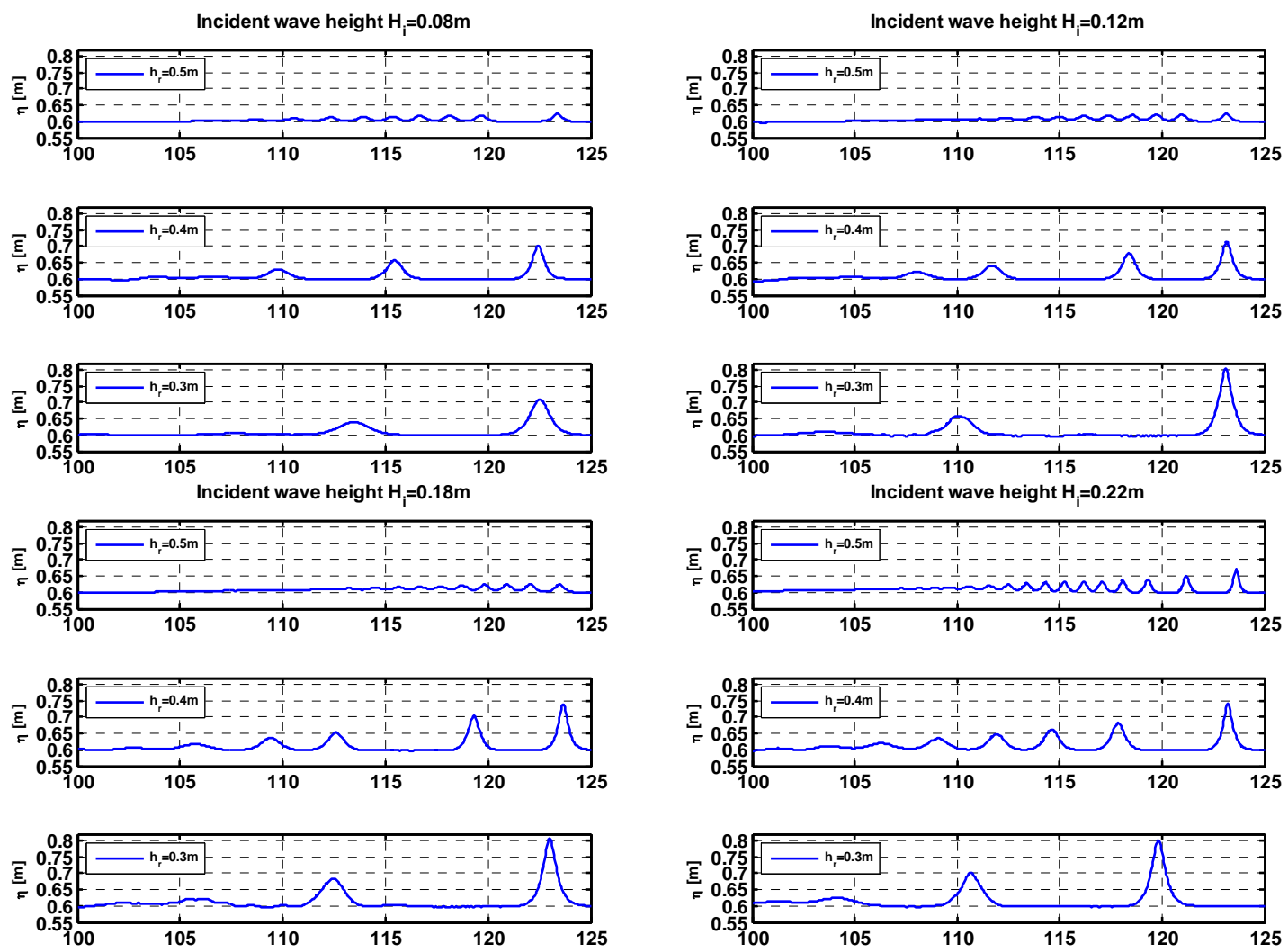

Figure 8. Transmitted wave trains at the end of the infinite reefs for all incident wave heights $H_{i}$ and all reef heights $d_{r}$.

\begin{tabular}{|c|c|c|c|c|c|c|c|c|}
\hline \multirow[b]{2}{*}{$h_{r}[\mathrm{~m}]$} & \multicolumn{2}{|c|}{$H_{i}=0.08 \mathrm{~m}$} & \multicolumn{2}{|c|}{$H_{i}=0.12 \mathrm{~m}$} & \multicolumn{2}{|c|}{$H_{i}=0.18 \mathrm{~m}$} & \multicolumn{2}{|c|}{$H_{i}=0.22 \mathrm{~m}$} \\
\hline & $\begin{array}{c}\text { visual } \\
\text { observation }\end{array}$ & NLFT & $\begin{array}{c}\text { visual } \\
\text { observation }\end{array}$ & NLFT & $\begin{array}{c}\text { visual } \\
\text { observation }\end{array}$ & NLFT & $\begin{array}{c}\text { visual } \\
\text { observation }\end{array}$ & NLFT \\
\hline 0.5 & 8 & 16 & 9 & 21 & 10 & 22 & 12 & 26 \\
\hline 0.4 & 5 & 7 & 5 & 7 & 6 & 8 & 7 & 8 \\
\hline 0.3 & 2 & 2 & $2(+1)$ & 3 & $2(+1)$ & 4 & $2(+1)$ & 4 \\
\hline
\end{tabular}

\section{Influence of reef width $\boldsymbol{b}_{r}$ on the number of transmitted solitons $\boldsymbol{N}_{\text {sol }}$}

The analysis of the influence of the wave height $H_{i}$ and the reef height $h_{r}$ showed that for high reefs, a turbulent bore is generated at the beginning of the reef (see Figure 1) that propagates over the reef. If the reef width is large enough, undular waves are generated at the front of the bore that will, if the reef width is long enough, evolve into a train of separated solitons. Table 3 shows the approximate propagation length over the reef i) until at least three undular bores are generated and ii) until the first soliton is separated from the bore. Generally, a reef width of at least $30 \mathrm{~m}$ is necessary for the first soliton to separate from the bore or the incident wave after it passed the reef front. Considering the incident wave lengths $L_{i}=6.97 \mathrm{~m}$ to $4.20 \mathrm{~m}$, for the required relative reef width applies $b_{r} / L_{i}=4.3$ to 7.1). This means that for the generation of a second soliton and depending on $H_{i}$, the reef width has to be at least more than four times the incident soliton wave length.

To obtain the number of transmitted solitons behind reefs with finite widths the wave trains behind reefs with $b_{r}=2.0 \mathrm{~m}, 10.0 \mathrm{~m}$ and $30.0 \mathrm{~m}$ are analysed at the end of the simulation area $(x=100 \mathrm{~m}$ $125 \mathrm{~m}$ for $b_{r}=2.0 \mathrm{~m}$ and $x=70 \mathrm{~m}-125 \mathrm{~m}$ for $b_{r}=10.0 \mathrm{~m}$ and $30.0 \mathrm{~m}$ ). The number of solitons from NLFT analysis is given in Table 4. In all analysed tests, only one or two transmitted solitons were found. Behind the reef with $b_{r}=2.0 \mathrm{~m}$ no more than one soliton was detected. This small reef width does not cause any bore generation or soliton fission. A maximum of two solitons are generated for 
higher $H_{i}$ and $h_{r}$. The analysis of samples with $b_{r}=50.0 \mathrm{~m}$ gave the same result: one soliton for $H_{i}=0.08 \mathrm{~m}$ and two solitons for the higher waves.

\begin{tabular}{|c|c|c|c|c|c|c|c|c|}
\hline \multirow{2}{*}{$h_{r}[\mathrm{~m}]$} & \multicolumn{2}{|c|}{$H_{i}=0.08 \mathrm{~m}$} & \multicolumn{2}{|c|}{$H_{i}=0.12 m$} & \multicolumn{2}{|c|}{$H_{i}=0.18 \mathrm{~m}$} & \multicolumn{2}{|c|}{$H_{i}=0.22 \mathrm{~m}$} \\
\hline & $3^{\text {rd }}$ bore & $1^{\text {st }}$ soliton & $3^{\text {rd }}$ bore & $1^{\text {st }}$ soliton & $3^{\text {rd }}$ bore & $1^{\text {st }}$ soliton & $3^{\text {rd }}$ bore & $1^{\text {st }}$ soliton \\
\hline 0.5 & 20 & 30 & 30 & 45 & 40 & 60 & 50 & 70 \\
\hline 0.4 & - & 25 & - & 30 & - & 50 & 35 & 50 \\
\hline 0.3 & - & 30 & - & 30 & - & 30 & - & 30 \\
\hline
\end{tabular}

Entry '-' means that no bore is generated.

\begin{tabular}{|c|c|c|c|c|c|c|c|c|c|c|c|c|}
\hline & \multicolumn{3}{|c|}{$H_{i}=0.08 \mathrm{~m}$} & \multicolumn{3}{|c|}{$H_{i}=0.12 \mathrm{~m}$} & \multicolumn{3}{|c|}{$H_{i}=0.18 \mathrm{~m}$} & \multicolumn{3}{|c|}{$H_{i}=0.22 \mathrm{~m}$} \\
\hline & \multicolumn{3}{|c|}{$b_{r}[-]$} & \multicolumn{3}{|c|}{$b_{r}[-]$} & \multicolumn{3}{|c|}{$b_{r}[-]$} & \multicolumn{3}{|c|}{$b_{r}[-]$} \\
\hline$h_{r}[\mathrm{~m}]$ & 2.0 & 10.0 & 30.0 & 2.0 & 10.0 & 30.0 & 2.0 & 10.0 & 30.0 & 2.0 & 10.0 & 30.0 \\
\hline 0.5 & 1 & 1 & 1 & 1 & 2 & 2 & 1 & 2 & 2 & 1 & 2 & 2 \\
\hline 0.4 & 1 & 1 & 2 & 1 & 1 & 2 & 1 & 2 & 2 & 1 & 2 & 2 \\
\hline 0.3 & 1 & 1 & 2 & 1 & 1 & 2 & 1 & 2 & 2 & 1 & 2 & 2 \\
\hline
\end{tabular}

Figure 9 shows the process of solitary wave propagation over a submerged narrow reef with $h_{r}=0.5 \mathrm{~m}$ and $b_{r}=2.0 \mathrm{~m}$. At $t=10 \mathrm{~s}$ the incident solitary wave is in front of the reef. At $t=18 \mathrm{~s}$ the wave passed the reef. In contrast to the processes in Figure 1 the shape of the soliton is conserved. Small oscillatory waves are generated behind the soliton. On the left side of the reef, the reflected soliton propagates toward the left boundary. The figure at $t=25 \mathrm{~s}$ shows the transmitted soliton, trailed by oscillatory waves which can clearly be identified by the fact that the wave profile passes the still water level. The reflected soliton reaches the boundary and will be reflected. At $t=35 \mathrm{~s}$ the transmitted waves are dispersed, but the type of the waves - leading soliton and trailing oscillatory waves - has not changed. The number of only one transmitted soliton is constant.

The analysis of the influence of the reef width $b_{r}$ has shown that very narrow reefs do not cause any bore generation or soliton fission. In the simulations, the minimum reef width at which a second soliton is generated is $b_{r}=10.0 \mathrm{~m}$. Increasing the reef width to $b_{r}=30.0 \mathrm{~m}$ or $50.0 \mathrm{~m}$ does not increase the maximum number of solitons.
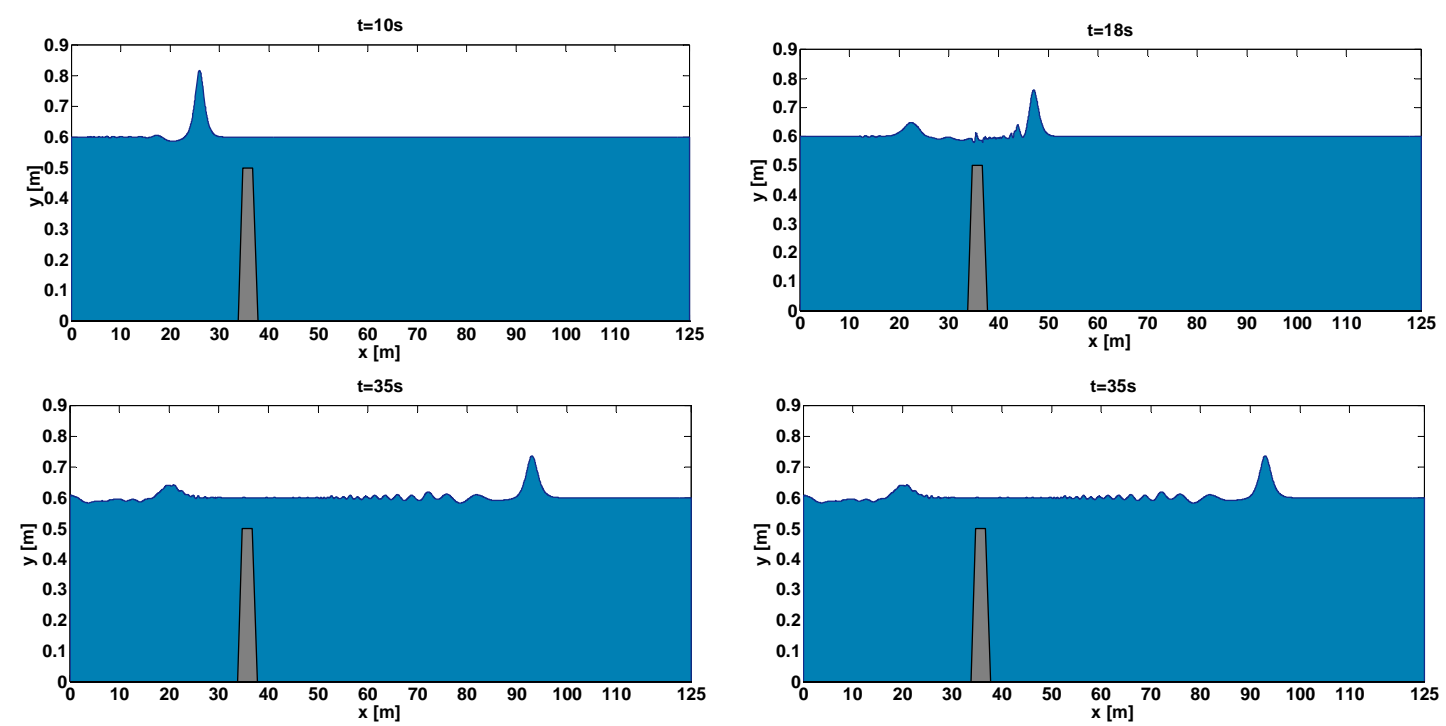

Figure 9. Transmission of a solitary wave $\left(H_{i}=0.22 \mathrm{~m}\right)$ over a submerged narrow reef $\left(h_{r}=0.5 \mathrm{~m}\right)$ with reef width $b_{r}=2.0 \mathrm{~m}$. 


\section{SUMMARY AND OUTLOOK}

Systematic numerical model tests using submerged reefs with "infinite" and finite width have been analysed using the Nonlinear Fourier Transform (NLFT). In the analysis of the obtained data from numerical simulations with high reef with infinite width the NLFT is able not only to determine the separated solitons in front of the undular bore. The nonlinear analysis algorithm also reliably provides the correct soliton wave heights of the undular waves in the bore. The maximum number of solitons $N_{s o l}$ provided by NLFT is larger than $N_{\text {sol }}$ from available approaches. The analysis of the data with bores leads to the generation of additional solitons in the NLFT. This problem is known and has to be considered during the interpretation of the analysis results. If the data do not contain bores, the NLFT can reliably quantify the correct number and height of the solitons.

The comparison of the simulation data with different incident wave height over an infinite reef shows that the number of separated solitons also depends on the incident wave height. The available design formulae for the number of solitons (Equations (1) - (3)) do not account for $H_{i}$.

The observations and analyses described here confirm the strong influence of $d_{r}$ on the number of transmitted solitons. The results clearly show, that the physical processes over the reef and the process of bore generation change if the reef height fall below a certain value. For lower reefs with $d_{r} / h \leq 0.33$, no undular bore is generated and the incident soliton disintegrates into several solitons directly at the beginning of the reef without forming a turbulent bore.

The analysis of the influence of the reef width $b_{r}$ has shown that very narrow reefs with $b_{r}=2,0 \mathrm{~m}$ $\left(b_{r} / L_{i}=0.3\right.$ to 0.5$)$ do not cause any bore generation or soliton fission. The maximum number of solitons behind the reef is one. In the simulations, the minimum reef width at which a second soliton is generated is $b_{r}=10.0 \mathrm{~m}\left(b_{r} / L_{i}=1.4\right.$ to 2.4$)$. Increasing the reef width to $b_{r}=30.0 \mathrm{~m}$ or $50.0 \mathrm{~m}$ $\left(b_{r} / L_{i}=4.3\right.$ to 7.1$)$ does not increase the maximum number of solitons. Madsen et al. (2008) stated that solitons "may steepen and disintegrate into an undular bore" when they approach the coast. But they have seen "no evidence that this can develop into a leading soliton". The analyses show that the bore has to propagate at least 4.3 to 7.1 times the incident solitary wave length before the first soliton will be fully dispersed from the bore. Considering the prototype length of a real tsunami, this means that soliton fisson is "not relevant for geophysical tsunamis" (Madsen et al., 2008).

Nevertheless, the analysis of soliton fission well illustrates the capability of NLFT for the analysis of nonlinear solitary waves. This method explicitly considers solitons and cnoidal waves as basic components for the analysis. The NLFT reliably identifies solitons and undular bores from data of incident solitary waves and transmitted wave trains. The analysis results have been confirmed by available formulae for infinite reefs and by visual observation of the analysed wave trains. The NLFT is able to identify solitons from undular bores even if the solitons have not yet fully dispersed from the bore. The NLFT has proved to be a powerful advanced analysis method for the analysis of nonlinear waves.

In the analyses presented here the local processes at the beginning of the reef, such as the type of wave breaking, is assumed to be negligible for the number of transmitted solitons behind the reefs. But the analyses showed clearly that the generation of the undular bore, that strongly affects the soliton fission and the number of solitons, depends on the reef height. For further detailed analyses the type of wave breaking should also be considered.

\section{ACKNOWLEDGMENTS}

This research project is supported by the German Research Foundation (DFG 1/9-1 and 1/9-2). The help of Prof. I.J. Losada (University of Cantabria, Santander, Spain) for providing COBRAS-UC and introducing the first author in the code is gratefully acknowledged.

\section{REFERENCES}

Ablowitz, M.J.; Kaup, D.J.; Newell, A.C.; Segur, H. (1973): Nonlinear-evolution equations of physical significance. Physical Review Letters, 31, 2, pp. 125-127.

Ablowitz, M.J.; Kaup, D.J.; Newell, A.C.; Segur, H. (1974): The Inverse Scattering Transform Fourier Analysis for Nonlinear Problems. Stud. Appl. Math., 53, 4, pp. 249-315.

Bleck, M.; Oumeraci, H. (2002): Hydraulic performance of artificial reefs: global and local description. Proceedings 28th International Conference Coastal Engineering (ICCE), ASCE, Volume 2, Cardiff, U.K., pp. 1778-1790. 
Lin, P. (2004): A numerical study of solitary wave interaction with rectangular obstacles. Coastal Engineering, Vol. 51, pp. 35-51.

Losada, M.A.; Vidal, C.; Median R. (1989): Experimental study on the evolution of a solitary wave at an abrupt junction. Journal of Geophysical Research, Vol. 94, No. C10, pp. 14557-14566.

Madsen, O.S.; Mei C.C. (1969): The transformation of a solitary wave over an uneven bottom. Journal of Fluid Mechanics, Vol. 39, Part 4, pp. 781-791.

Madsen, P.A.; Fuhrmann, D.R.; Schäffer, H.A. (2008): A critical discussion of the solitary wave paradigm for tsunamis. Proceedings 31st International Conference Coastal Engineering (ICCE), ASCE, Volume 2, Hamburg, Germany pp. 1262-1274.

Osborne, A.R. (1991): Nonlinear Fourier Analysis for the Infinite-Interval Korteweg-de Vries Equation I: An Algorithm for the Direct Scattering Transform. Journal of Computational Physics, 94, 284-313.

Provenzale, A.; Osborne, A.R. (1991): Nonlinear Fourier Analysis for the Infinite-Interval Kortewegde Vries Equation II: Numerical Tests of Direct Scattering Transform. Journal of Computational Physics, 94, 314-351.

Seabra-Santos F.J.; Renouard D.P.; Temperville A.M. (1987): Numerical and experimental study of the transformation of a solitary wave over a shelf or isolated obstacle. Journal of Fluid Mechanics, Vol. 176, pp.117-134.

Strusinska, A.; Oumeraci, H. (2008): Hydraulic performance of artificial reef for tsunami protection, Report No. 3: Laboratory experiments on solitary wave nonlinear transformation over a submerged structure of a finite width. Leichtweiß-Institute for Hydraulic Engineering and Water Resources, Dept. of Hydromechanics and Coastal Engineering, Technische Universität Braunschweig, Braunschweig, 241 pp. 